\title{
Effect of bowel cleansing on colonic transit in constipation due to slow transit or evacuation disorder
}

\author{
C. E. J. SLOOTS ${ }^{\star} \dagger \&$ R. J. F. FELT-BERSMA ${ }^{\star} \dagger$ \\ ${ }^{\star}$ Department of Gastroenterology of the 'Vrije Universiteit' Medical Centre, Amsterdam, The Netherlands \\ $\dagger$ Department of Gastroenterology and Hepatology, Erasmus Medical Centre, Rotterdam, The Netherlands
}

\begin{abstract}
Colon transit time measurement with radioopaque markers is a method of studying the passage of luminal contents throughout the colon. Overall colonic transit time (CTT), as well as segmental transit times [right (RTT), left (LTT) and rectosigmoid (RSTT)], can be calculated. We hypothesize that CTT is influenced by faecal impaction when the rectum is emptied infrequently. The aim of this study is to investigate the effect of bowel cleansing on colonic transit time in patients with chronic constipation. In 25 women (age 41 years; range 20-65 years) with constipation according to Thompson criteria, CTT measurement was performed in an unprepared situation and repeated after cleansing with 4 L of Klean-Prep ${ }^{\circledR}$. Ten healthy female volunteers (age 41 years; range 27-57 years) were used as controls. In constipated patients, CTT decreased from a median $70 \mathrm{~h}$ (range 10-130 h) to $48 \mathrm{~h}$ (5-94 h) in the cleansed state $(P<0.001)$. A shortening of transit time was found in all three segments. In 10 patients with slow transit (ST) (CTT > $86 \mathrm{~h})$, CTT decreased from $110 \mathrm{~h}$ (range 94-130) to 86 (38-94) $(P<0.001)$. Five of the 10 patients with $S T$ before bowel cleansing had a CTT below $86 \mathrm{~h}$ after cleansing. In female controls, uncleansed CTT and RSTT shortened from $39 \mathrm{~h}(23$ to 62) and $17 \mathrm{~h}(8-29)$ to $29 \mathrm{~h}(17-48)$ and $10 \mathrm{~h}(0-20)$ after bowel cleansing $(P=0.058$ and $P=0.046)$. Colonic intraluminal contents have a substantial effect on colonic transit. In female controls, bowel cleansing shortened rectosigmoid transit. Women with constipation had faster transit in the cleansed state, however, the distribution of markers was not altered. Despite the effect of bowel
\end{abstract}

Address for correspondence

R. J. F. Felt-Bersma, Department of Gastroenterology and Hepatology, Erasmus Medical Centre Rotterdam, PO Box 2040, 3000 CA Rotterdam, The Netherlands.

Tel.: + 3110 4635946; fax: + 3110 4634682;

e-mail: felt@mdl.azr.nl

Received: 17 May 2001

Accepted for publication: 7 September 2001 cleansing on CTT, it seems unnecessary to prepare the bowel in clinical practice because the differentiation of patients between slow transit constipation and outlet obstruction is not changed. However, because in an infrequent defecation pattern, the influence of faecal impaction is considerable, CTT should be applied with care for critical clinical decisions in the treatment of constipation.

Keywords colonic transit time, constipation, radioopaque marker technique.

\section{INTRODUCTION}

Colonic transit time (CTT) measurement with the use of radio-opaque markers is a method for investigating the passage of colonic intraluminal contents throughout the colon. CTT correlates with the pattern of defecation ${ }^{1-3}$ and is comparable to the transit time measured with scintigraphic studies. ${ }^{1,4}$ Overall colonic transit time can be measured by counting the radioopaque markers on the plain abdominal radiograph. Patients are categorized as having 'slow transit constipation' if overall colonic transit time is delayed. Segmental transit times can be calculated after counting the markers in the colonic regions. Different methods are used to describe the distribution of the radio-opaque markers on the radiograph. The most practical subdivision is into three segments: right-sided area, left-sided area and rectosigmoid area. ${ }^{5}$ With this subdivision, it is possible to identify an accumulation of markers in the rectosigmoid region, which could direct to an 'outlet obstruction' ${ }^{6-8}$ CTT measurement with radio-opaque markers is progressively used in clinical practice because it is easy to perform with little patient inconvenience. CTT objectifies the complaints of patients with constipation, ${ }^{9}$ and is a useful criterion for selection of patients for surgery ${ }^{10,11}$ and for the evaluation of new entero-kinetic drugs. ${ }^{12,13}$ 
Few data are available on the influence of faecal impaction on CTT. The CTT is influenced substantially in an irregular defecation pattern when healthy subjects willingly retain stools. Mainly rectosigmoid transit and, by reflex mediation, right colonic transit is increased. ${ }^{14}$ In constipated patients, the distribution of markers is altered when CTT is measured after bowel preparation with a light laxative. ${ }^{15}$ It is not clear if delayed colonic transit in patients with obstructive defecation is attributable to retained faeces in the rectum, or to a coexistent disorder of colonic motor function. We hypothesized that colonic cleansing would normalize colonic transit when the latter was attributable to retained faeces in the rectum, as opposed to a colonic motility disorder. Therefore, we measured colonic transit in an uncleansed and in a cleansed state both in patients with constipation and in healthy females.

\section{PATIENTS AND METHODS}

\section{Subjects}

Twenty-five consecutive female patients (age 41 years; range 20-65 years) were included for experiment 1 . All had constipation according to the Thompson criteria ${ }^{16}$ consisting of two of the following criteria for at least 6 months: less than two spontaneous bowel movements in a week (without laxatives) or $25 \%$ hard stools or $25 \%$ sensation of incomplete evacuation or $25 \%$ straining. None of the patients had previous abdominal surgery other than a hysterectomy (in seven). All patients used laxatives.

Ten healthy females (median age 41 years; range 27 57 years) were recruited by advertising for experiment 2. The participants had normal bowel habits without history of constipation or abdominal surgery. The volunteers did not use medication. Females who participated in the study were not pregnant. The protocol was approved by the local Ethics Committee of the Vrije Universiteit Medical Centre and written informed consent was given by each subject.

\section{Study design}

Experiment 1. Patients with constipation ingested a capsule with 10 radio-opaque markers at $8.00 \mathrm{~h}$ daily on six consecutive days. On the seventh day, a plain abdominal radiograph was performed. After a washout period of 10 days, $4 \mathrm{~L}$ of Klean-Prep ${ }^{\circledR}$ was ingested the day before the subjects took the first of the markers. The subjects again ingested the markers on six consecutive days and a plain abdominal radiograph was performed on the seventh day.
Experiment 2. The subjects ingested two capsules with 10 radio-opaque markers, at $8.00 \mathrm{~h}$ and at $20.00 \mathrm{~h}$ daily for three consecutive days. On the fourth day a plain abdominal radiograph was performed. After a washout period of 10 days, $4 \mathrm{~L}$ of Klean-Prep ${ }^{\circledR}$ was ingested the day before the subjects took the first set of markers for the second colonic transit time measurement. Throughout the study period, the volunteers were asked to register the defecation frequency and stool consistency in a diary.

\section{Colonic transit time measurement}

Colonic transit time (CTT) measurement was assessed by means of a modified radio-opaque marker technique using gelatine capsules containing 10 radio-opaque polyurethane markers consisting of $40 \%$ barium sulphate (P. \& A. Mauch, Münchenstein, Switzerland). Overall CTT was calculated by counting the total number of markers on the plain abdominal radiograph. Segmental CTT was calculated using the number of markers in the three segments identified according to Arhan et al. ${ }^{5}$ The central point was the fifth lumbar vertebra. The right colonic segment was the part between the line over the right pelvic outlet ring and the line over the spinal processes of the lumbar vertebra. The left colonic segment was the part between the line over the spinal processes of the lumbar vertebra and the left pelvic rim, the distal part was the rectosigmoid area. In the determination of the segments, the configuration of the air in the bowel was taken into account.

To calculate the transit time, the modified Metcalf formula was used. ${ }^{17}$ As the subjects ingested 10 radio-opaque markers daily for 6 days in experiment 1 and 10 markers, twice daily, for 3 days in experiment 2 , the overall colonic transit time was calculated accordingly:

$$
\mathrm{CTT}=1 / N * \sum_{\mathrm{i}=1}^{\mathrm{i}} N_{\mathrm{i}}\left[1 / 2\left(\mathrm{t}_{\mathrm{i}+1}-\mathrm{t}_{\mathrm{i}-1}\right)\right]
$$

where $\mathrm{CT}=$ overall colonic transit time; $N=$ total number of a particular markers given; $i=$ number of capsules taken; $N_{\mathrm{I}}=$ number of markers of a particular type present on the film; and $1 / 2\left(t_{i}+1-t_{i}-1\right)=$ time interval between successive intakes of markers.

This formula was summarized in the 6-day method (patients):

$$
\mathrm{CTT}=2.4 \times N_{\mathrm{i}}(\text { in hours })
$$

and in the 3-day method (controls):

$$
\mathrm{CTT}=1.2 \times N_{\mathrm{i}} \text { (in hours) }
$$

Segmental transit time was calculated in a similar way to the overall CTT. Slow transit constipation was 
defined as more than $86 \mathrm{~h}$, which is the upper limit of the 95th percentile of the CTT in 84 females using the same method. ${ }^{18}$ Outlet obstruction was defined as more than $50 \%$ of the markers in the rectosigmoid area. ${ }^{15}$

\section{Bowel cleansing}

For bowel cleansing, $4 \mathrm{~L}$ of the osmotic laxative Klean-prep ${ }^{\circledR}$ was ingested within 4-6 h. One litre of Klean-prep ${ }^{\circledR}$ contained $17.6 \mathrm{mmol}(59 \mathrm{~g})$ macrogol 3350 (polyethylene glycol), $125 \mathrm{mmol}$ sodium, $10 \mathrm{mmol}$ potassium, $40 \mathrm{mmol}$ sulphate, $35 \mathrm{mmol}$ chloride, $20 \mathrm{mmol}$ hydroxycarbonate and $49.4 \mathrm{mg}$ aspartame. All healthy subjects produced clear fluids after cleansing. All patients with constipation produced fluids after intake of the solution; however, not all had clear fluids. The bowel cleansing solution was well tolerated by all subjects.

\section{Anorectal function}

The maximal basal pressure was measured according to our methods as described previously. ${ }^{19}$ To determine relaxation of the pelvic floor, the patients were asked to strain with the catheter in situ. ${ }^{20,21}$ Pelvic floor dyssynergia was defined as a maximal basal pressure of more than $60 \mathrm{mmHg}$ and paradoxical increase of anal pressure during straining of more than $10 \mathrm{mmHg}$.

\section{Data analysis and statistics}

Results are presented as median and range because the distribution of the data was nonparametric. MannWhitney $U$-test was used to compare data in normal situation. Wilcoxon paired signed ranks test was used to compare data in the basal situation and after bowel cleansing.

\section{RESULTS}

\section{Healthy females}

Throughout the study period, reported stool consistency and frequency remained constant. Loose stools and an increased frequency were only reported during the use of the cleansing solution until the following evening. The first day after the cleansing, stools normalized.

In the uncleansed state, overall CTT and rectosigmoid transit time (RSTT) were $39 \mathrm{~h}$ (range 3-62 h) and $17 \mathrm{~h}(8-29 \mathrm{~h})$. After bowel cleansing, RSTT decreased to $10 \mathrm{~h}(0-20 \mathrm{~h})(P=0.046)$ and overall
Figure 1 (a-d) Colonic transit time in females in the uncleansed situation and after bowel cleansing. Median values are plotted as bold lines.
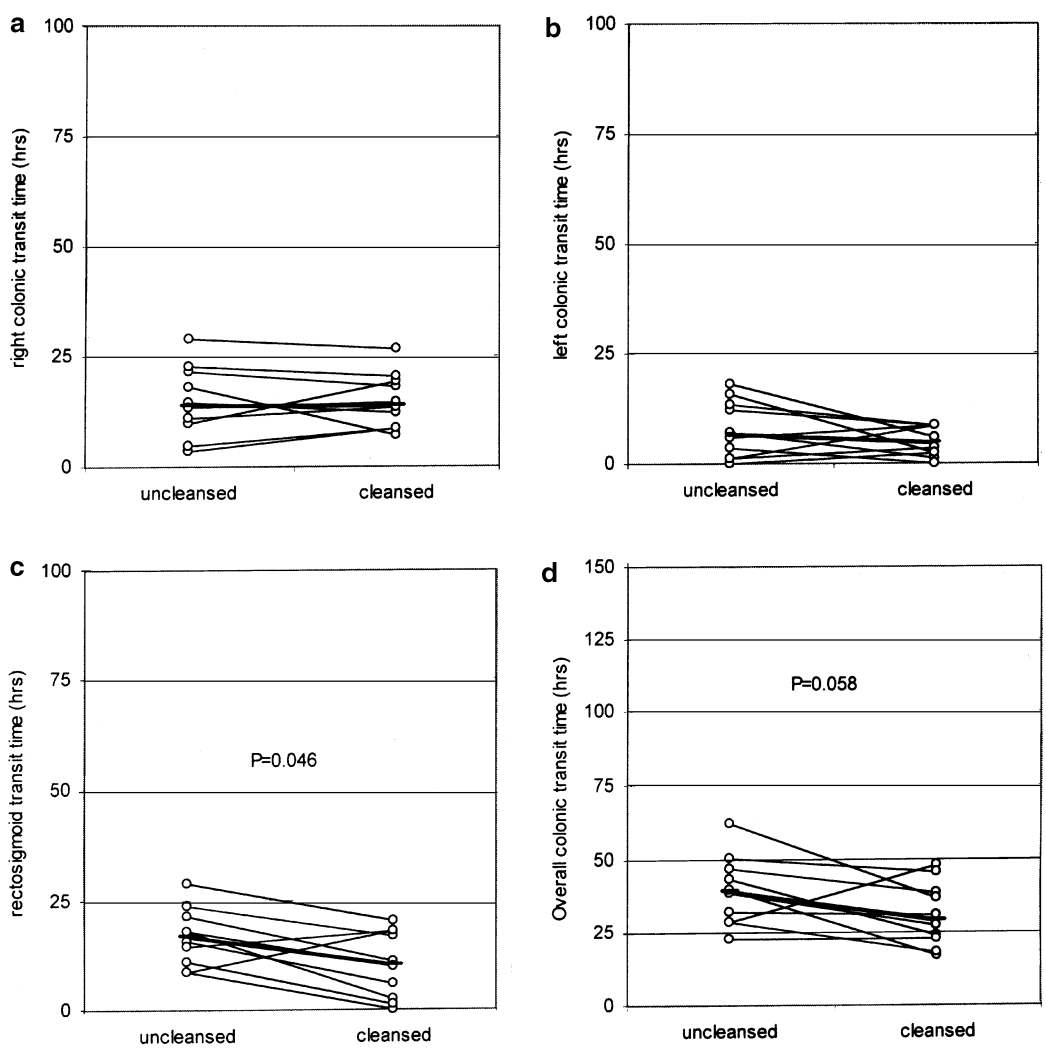

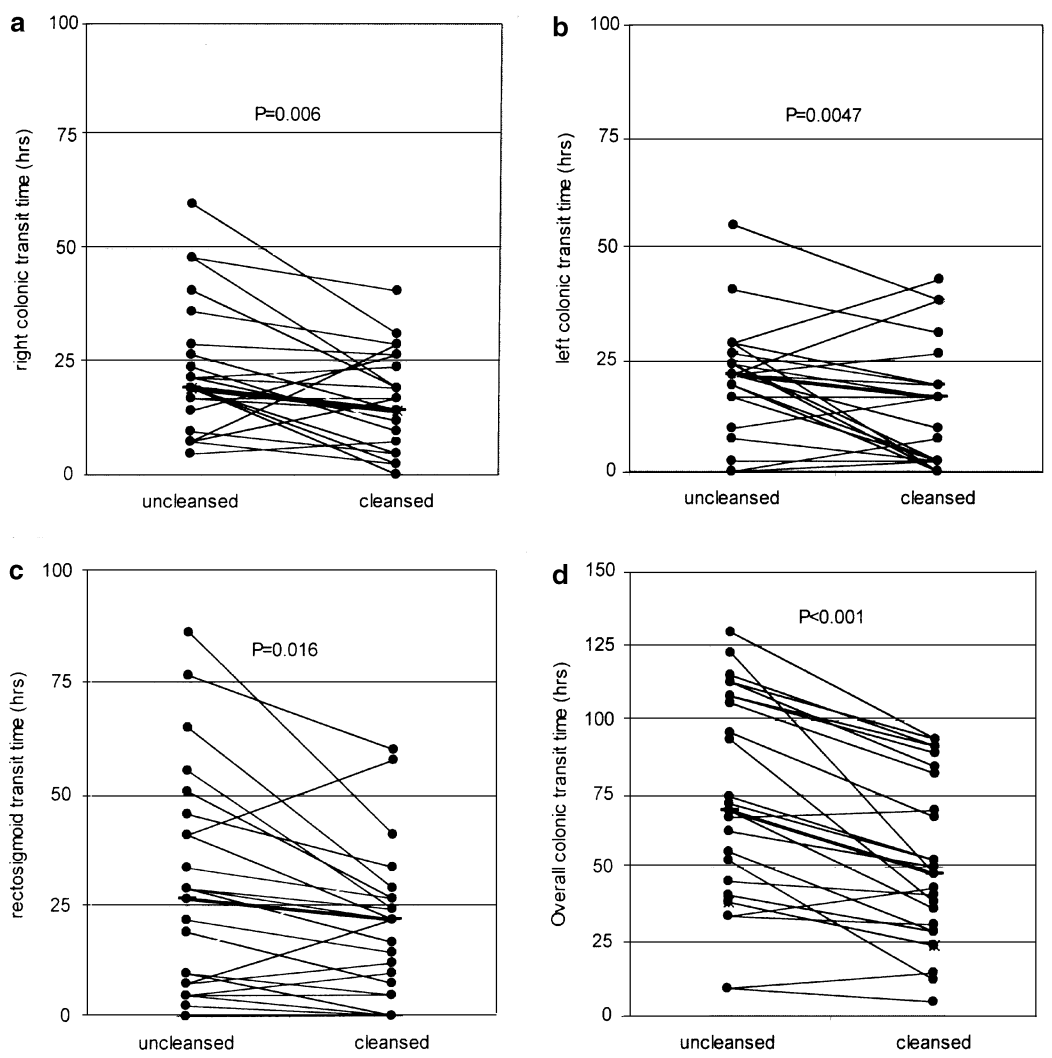

Figure 2 (a-d) Colonic transit time in constipated patients, uncleansed and after bowel cleansing. Median values are plotted as bold lines.
CTT tended to decrease to $29 \mathrm{~h}(17-48 \mathrm{~h})(P=0.058)$. In only one of the 10 subjects was an increase in overall CTT found after cleansing (Fig. 1a-d).

\section{Patients with constipation}

In the uncleansed state overall colonic transit time was $70 \mathrm{~h}(10-130$ h) (Fig. 2a-d). Slow transit (ST) was found in 10 patients and 15 patients had a transit within the normal range. Overall colon transit time after bowel cleansing was significantly shorter (48 h; range 5-94 h; $P<0.001)$ than in the uncleansed state. A small increase of overall CTT in the cleansed state was found in $3 / 25$ patients. A decrease in transit was found in all colonic segments. The overall colonic transit time in ST patients decreased from 110 to $86 \mathrm{~h}(P=0.005)$. This difference in CTT was larger in ST patients than in normal transit patients $(-26 \mathrm{~h} v s .-12 \mathrm{~h} ; P=0.004)$. Five ST patients in the uncleansed situation had a transit time within the normal range after bowel cleansing. However, when the upper limit of the range of cleansed CTT in the female controls (more than $48 \mathrm{~h}$ ) was applied to form the criterion for ST, only one patient with ST in the uncleansed state did not have ST in the cleansed state. Two patients without ST in the uncleansed state had ST in the cleansed state. In our group, six patients met the outlet obstruction criteria in the unprepared CTT. However, in the cleansed state, five of six patients still fulfilled this criterion and one new patient could be added.

\section{Pelvic floor dyssynergia}

Ten patients had manometric signs of pelvic floor dyssynergia. Of these patients, one had outlet obstruction, one had slow transit and two had combined slow transit and an outlet obstruction (> 50\% of the markers in the rectosigmoid region). RSTT was not significantly different between the patients with pelvic floor dyssynergia (20.4 h; range $2.4-86 \mathrm{~h}$ ) and the patients without pelvic floor dyssynergia $(28.8 \mathrm{~h}$; range 0-65 h). Overall CTT in the uncleansed state was $61 \mathrm{~h}(10-130 \mathrm{~h})$, which shortened in the cleansed state to $38 \mathrm{~h}(12-94 \mathrm{~h})(P=0.001)$. RSTT decreased to $18 \mathrm{~h}(0-60 \mathrm{~h})$ after cleansing $(P=0.059)$. The distribution of the markers was not altered. After cleansing, the proportion of patients with outlet obstruction was unaltered. One patient who had slow transit in the uncleansed state $(122 \mathrm{~h})$, had a dramatically decreased overall transit time after cleansing (48 h) and in one patient, transit time $(67 \mathrm{~h})$ did not change after cleansing. 


\section{DISCUSSION}

This study was performed to confirm the hypothesis that fluctuations in the filling state of the colon substantially influence CTT measurement. As strict clinical conclusions are drawn from CTT, it is important to know whether CTT can differ after, for example, a very large bowel movement occurring once every week, which is often claimed by patients with constipation. Furthermore, it is not clear if delayed colonic transit in patients with obstructive defecation is attributable to retained faeces in the rectum, or to a coexistent disorder of colonic motor function. We hypothesized that colonic cleansing would normalize colonic transit when the latter was attributable to retained faeces in the rectum, as opposed to a colonic motility disorder. Our study showed that bowel cleansing decreased CTT substantially in constipated patients. In $50 \%$ of the patients with slow transit, transit time decreased to the normal range in the cleansed state. However, cleansing the bowel in controls decreased rectosigmoid transit time significantly and a trend was found in a decrease of overall CTT. Therefore, we conclude that the upper limit of the cleansed CTT values in controls (e.g. $48 \mathrm{~h}$ ) should be used to determine the cut-off point for detection of slow transit. Only one patient who had slow transit in the uncleansed state $(>86 \mathrm{~h})$, had transit within the normal range for the cleansed state $(<48 \mathrm{~h}$ ). Therefore, if slow transit was found with a CTT measurement in an uncleansed state, this was mainly due to decreased colonic function. Faecal impaction did play a role in delaying CTT, but when this impaction was resolved, delay in transit was still found.

Faecal impaction in the distal colon would be expected in patients with pelvic floor dyssynergia. Pelvic floor dyssynergia patients are unable to expel stools through paradoxical sphincter contraction and thus are troubled by obstructive defecation. ${ }^{20}$ In agreement with other studies, ${ }^{22}$ we found that in these patients rectosigmoid delay (outlet obstruction) in the uncleansed state was not more usual than in patients without pelvic floor dyssynergia. Furthermore, the overall transit time was shortened in the cleansed state, but the rectosigmoid transit was not particularly decreased. We expected that slow transit would be secondary to faecal impaction in an outlet disorder and that the true level of obstruction would be found after washing-out the faecal mass. We could not confirm this in our study.

Some points have to be made on the definition of pelvic floor dyssynergia. Rao et al. ${ }^{23}$ showed that paradoxical anal contraction occurs in subjects without constipation. Ten of 45 healthy subjects showed an obstructive pattern during anorectal manometry and four were unable to expel a $50-\mathrm{mL}$ water-filled balloon. Voderholzer et al. ${ }^{24}$ evaluated paradoxical sphincter contraction (PSC) in patients with constipation, patients with incontinence and controls using digital examination, anal manometry, defecography and radioopaque marker transit measurement. In the patients, $41 \%$ of the constipated and $25 \%$ of the incontinent patients showed manometric paradoxical sphincter contraction, as did $22 \%$ of the controls. The overlap of manometry, digital examination and defecography was very small $(5 \%)$. It was concluded that PSC is primarily a laboratory artefact. ${ }^{22}$ However, in patients with incontinence, the paradoxical increase during straining is a defensive mechanism to prevent loosing stools or air unwillingly. Controls can be embarrassed by the method of investigation.

Only one study has been published about bowel preparation and colonic transit time. Bergin and Read ${ }^{15}$ performed a study in 25 constipated patients $(22 \mathrm{~F}, 3 \mathrm{M})$ in whom colonic transit time was measured unprepared and 3 days after purging with $20 \mathrm{mg}$ picosulphate. The overall CTT was unchanged, but the distribution of the markers in the colon was altered; the markers accumulated more distally and were suggestive for an outlet obstruction. The authors concluded that the accumulation of faeces in the rectum under normal conditions caused a distribution pattern similar to colon inertia. Removal of this faecal 'mass' enabled the markers to progress to the true obstruction. ${ }^{15}$ In contrast, we did not find different distribution patterns of the markers. Our patients had lower transit times in the cleansed state but the distribution appeared to be similar. There were some differences between the two studies. Firstly, we used bowel cleansing in order to empty the colon rigorously before measuring the second CTT. Secondly, the subjects started directly with the marker intake the day after the bowel cleansing. Finally, we studied both constipated patients with 'normal' CTT as well as with slow transit.

In all healthy subjects, $4 \mathrm{~L}$ of Klean-Prep ${ }^{\circledR}$ cleared the colon of faeces and resulted in watery stools. The first day after bowel cleansing, stools normalized. On the days in which patients took the capsules, they produced normal stools, therefore a direct effect of the laxative on the CTT measurement seems unlikely. Klean-prep ${ }^{\circledR}$ was not always able to clean the bowel fully in constipated patients; some reported they still had coloured but watery stools. However, they all passed a large quantity of stools and faecal impaction was absent. The production of watery stools stopped 
after 1 day and a direct effect of the cleansing on the CTT is unlikely.

Various methods are described for measurement of CTT. Single ${ }^{5,15}$ or multiple markers ${ }^{24}$ are used with one $\mathrm{e}^{15,18}$ or more abdominal radiographs at different intervals. ${ }^{5,8,17}$ Methods over $3^{8,15,17,24}$ or $6^{6,18}$ days have been performed and different formulae have been used to calculate the transit time, ${ }^{25}$ even different lines to determine the segments. ${ }^{5,15,26}$ Therefore, normal values (upper limit of $95 \%$ confidence interval) range from 68 to $113 \mathrm{~h}$ in the literature. ${ }^{6,8,17}$ We used a 6-day method with a single marker and single radiograph technique to study constipated patients. The upper limit of the range of uncleansed CTT in female controls was $62 \mathrm{~h}$ in our study. However, the definition of slow transit constipation (more than $86 \mathrm{~h}$ ) was used according to Meier et al. who studied 86 females using the same method. ${ }^{18}$ Outlet obstruction was defined as more than $50 \%$ of the markers in the rectosigmoid area according to Bergin and Read. ${ }^{15}$ We used a 3-day method in controls because we expected that the CTT would be shortened to less than $24 \mathrm{~h}$ and thus most markers would be expelled before the radiograph was taken. The calculation of CTT would be less accurate if a 6-day method was used. ${ }^{12}$

In the interpretation of the results of this study, we presumed that CTT with the use of radio-opaque markers is reproducible in constipated patients. However, to our knowledge, a reproducibility study using an adequate analysis has never been published. Bouchoucha et al. ${ }^{8}$ explored reproducibility in 28 patients with irritable bowel syndrome who twice underwent a CTT measurement, each a month apart. Results of the two measurements were found to be similar, with correlation coefficients in the range of $r=0.6$. However, in this comparative study, two different methods of CTT were used (multiple markersingle X-ray and single marker-multiple X-ray) and subjects were patients with irritable bowel syndrome, not patients with constipation. ${ }^{8}$ Knowles et al. ${ }^{27}$ performed colon transit time measurement in 16 irritable bowel syndrome (IBS) patients a month apart during placebo treatment in a trial. Test-retest reliability was good for overall colonic transit time $(r=0.71)$, but was lower for segmental transit time (ranging from $r=0.5$ for RTT and LTT to 0.3 for RSTT). In patients with an irregular defecation pattern, CTT measurement may be less reproducible as this test is influenced by several factors such as intrasubject variability in defecation pattern and the relative short period of measurement. However, such a reproducibility study still has to be performed in constipated patients.
In conclusion, colonic intraluminal contents have a substantial effect on colonic transit. In female controls, bowel cleansing shortened colonic transit time, and this was most pronounced in the rectosigmoid area. Women with constipation had faster transit in the cleansed state, but the distribution of markers was not changed. Despite the effect of bowel cleansing on CTT, it seems unnecessary to prepare the bowel in clinical practice because the differentiation of patients between slow transit constipation and outlet obstruction is not changed. Because in an infrequent defecation pattern the influence of faecal impaction is considerable, results of CTT measurement should be applied with care when used in critical clinical decisions for the treatment of constipation.

\section{REFERENCES}

1 Degen LP, Phillips SF. How well does stool form reflect colonic transit? Gut 1996; 3: 109-13.

2 Ashraf W, Park F, Lof J, Quigley EMM. An examination of the reliability of reported stool frequency in the diagnosis of idiopathic constipation. Am J Gastroenterol 1996; 91: 26-32.

3 Proano M, Camilleri M, Phillips SF, Thomforde GM, Brown ML, Tucker RL. Unprepared human colon does not discriminate between solids and liquids. Am I Physiol 1991; 260: G13-6.

4 Van der Sijp JR, Kamm MA, Nightingale JM et al. Radioisotope determination of regional colonic transit in severe constipation: comparision with radio-opaque markers. Gut 1993; 34: 402-8.

5 Arhan P, Devroede G, Jehannin B et al. Segmental colonic transit time. Dis Colon Rectum 1981; 24: 625-9.

6 Karlbohm U, Pahlman L, Nilsson S, Graf W. Relationships between defecographic findings, rectal emptying and colonic transit time in constipated patients. Gut 1995; 36: 907-12.

7 Karasick S, Ehrlich S. Is constipation a disorder of defection or impaired motility? Distinction based on defecography and colonic transit studies. AJR 1996; 166: 63-6.

8 Bouchoucha M, Devroede G, Arhan P et al. What is the meaning of colon transit time measurement? Dis Colon Rectum 1992; 35: 773-82.

9 American Gastroenterological Association. American Gastroenterological Association Medical Position Statement on Anorectal Testing Techniques. Gastroenterology 1999; 116: 732-60.

10 Pluta $\mathrm{H}$, Bowes KL, Jewell LD. Long-term results of total abdominal colectomy for chronic constipation: value of preoperative assessment. Dis Colon Rectum 1996; 39: 160-6.

11 Pezim ME, Pemberton JH, Levin KE, Litchy WJ, Phillips SF. Parameters of anorectal and colonic motility in health and in severe constipation. Dis Colon Rectum 1993; 36: 484-91.

12 Poen AC, Felt-Bersma RJF, van Dongen PAM, Meuwissen SGM. Effect of prucalopride, a new prokinetic agent, on 
gastrointestinal transit and anorectal function in healthy volunteers. Aliment Pharmacol Therap 1999; 13: 1493-7.

13 Houghton LA, Foster JM, Whorwell PJ. Alosetron: a 5-HT3 receptor antagonist, delays colonic transit in patients with irritable bowel syndrome and healthy volunteers. Aliment Pharmacol Ther 2000; 14: 775-82.

14 Klauseries AG, Voderholzer WA, Heinrich CA, Schindlbeck NE, Muller-Lissner SA. Behavioural modification of colonic function. Can constipation be learned? Dig Dis Sci 1990; 35/10: 1271-5.

15 Bergin AJ, Read NW. The effect of preliminary bowel preparation on a simple test of colonic transit in constipated subjects. Int I Colorectal Dis 1993; 8/2: 75-7.

16 Thompson WG, Heaton KW. Functional bowel disorders in apparently healthy people. Gastroenterology 1980; 79: 283-8.

17 Metcalf AM, Phillips SF, Zinsmeister AR, MacCarty RL, Beart RW, Wolff BG. Simplified assessment of segmental colonic transit. Gastroenterology 1987; 92/1: 40-7.

18 Meier R, Beglinger C, Dederding JP et al. Influence of age, gender, hormonal status and smoking habits on colonic transit time. Neurogastroenterol Motil 1995; 7: 235-8.

19 Poen AC, de Brauw M, Felt-Bersma RJ, de Jong D, Cuesta MA. Laparoscopic rectopexy for complete rectal prolapse. Clinical outcome and anorectal function tests. Surg Endosc 1996; 10: 904-8.
20 Preston DM, Lennard-Jones JE. Anismus in chronic constipation. Dig Dis Sci 1985; 30: 413-8.

21 Koutsomanis D, Lennard-Jones JE, Kamm MA. Prospective study of biofeedback treatment for patients with slow and normal transit constipation. Eur I Gastroenterol Hepatol 1994; 6: 141-7.

22 Voderholzer WA, Neuhaus DA, KlauSeries AG, Tzavella K, Müller-Lissner SA, Schindlbeck NE. Paradoxical sphincter contraction is rarely indicative of anismus. Gut 1997; 41: 258-62.

23 Rao SS, Hatfield R, Soffer E, Rao S, Beaty J, Conklin JL. Manometric tests of anorectal function in healthy adults. Am I Gastroenterol 1999; 94: 773-83.

24 Corazziari E, Badiali D, Habib FI et al. Small volume isosmotic polyethylene glycol electrolyte balanced solution (PMF-100) in treatment of chronic nonorganic constipation. Dig Dis Sci 1996; 41: 1636-42.

25 Bouchoucha M, Thomas SR. Error analysis of classic colonic transit time estimates. Am I Physiol Gastrointest Liver Physiol 2000; 279: G520-7.

26 Rausch T, Beglinger C, Alam N, Gyr K, Meier R. Effect of transdermal application of nicotine on colonic transit in healthy nonsmoking volunteers. Neurogastroenterol Motil 1998; 10: 263-70.

27 Knowles JB, Whitehead WE, Meyer KB. Reliability of a modified Sitzmark study of whole gut transit time. Gastroenterology 1998; : A779. 